This item was submitted to Loughborough's Research Repository by the author.

Items in Figshare are protected by copyright, with all rights reserved, unless otherwise indicated.

\title{
An asymmetric synthesis of trans-fused butyrolactones from endoperoxides
}

PLEASE CITE THE PUBLISHED VERSION

http://dx.doi.org/10.1021/jo400177j

\section{PUBLISHER}

(c) American Chemical Society

\section{VERSION}

AM (Accepted Manuscript)

\section{LICENCE}

CC BY-NC-ND 4.0

\section{REPOSITORY RECORD}

Priest, Joshua, Mark R. Longland, Mark R.J. Elsegood, and Marc C. Kimber. 2019. "An Asymmetric Synthesis of Trans-fused Butyrolactones from Endoperoxides”. figshare. https://hdl.handle.net/2134/13934. 
This item was submitted to Loughborough's Institutional Repository (https://dspace.lboro.ac.uk/) by the author and is made available under the following Creative Commons Licence conditions.

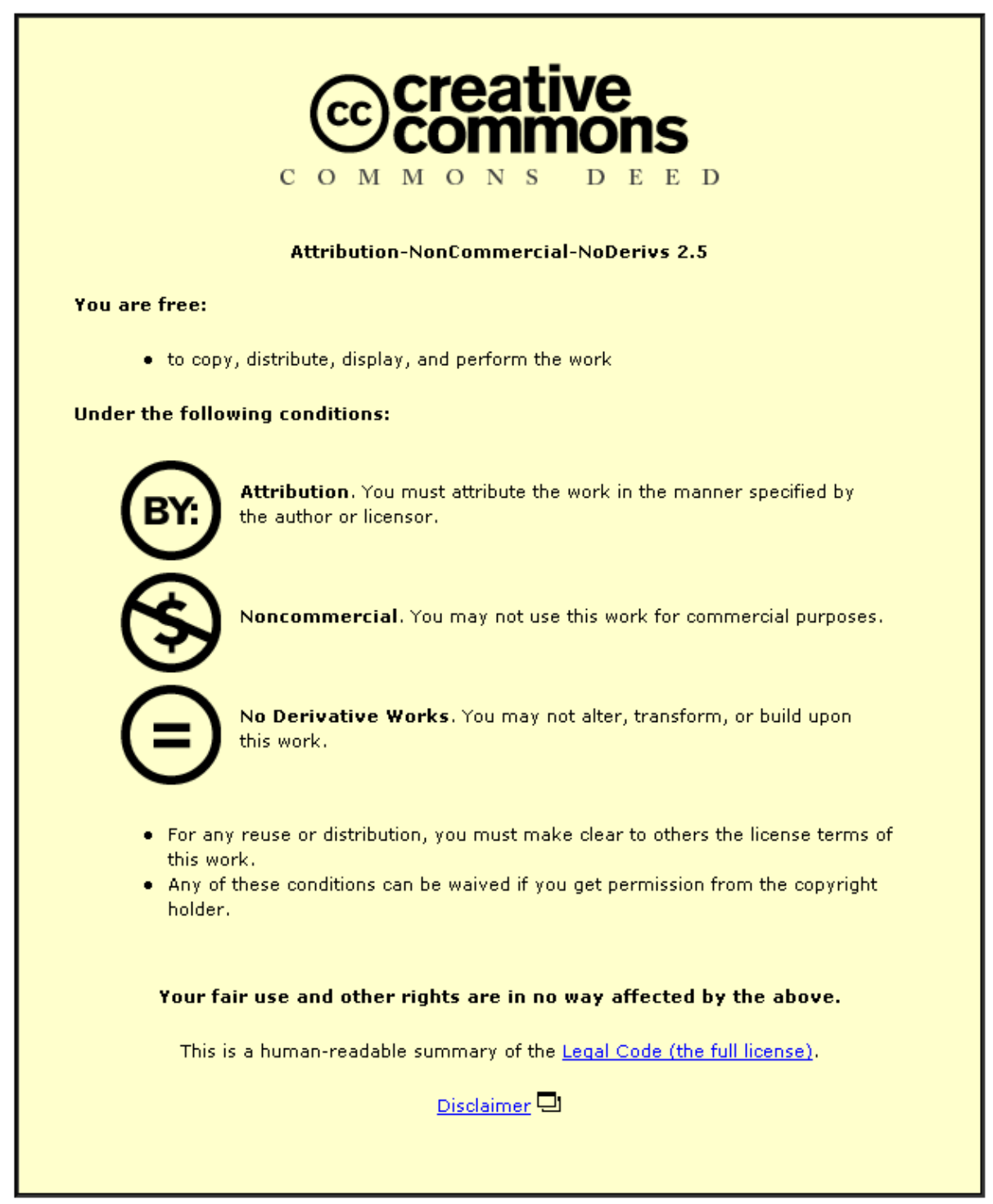

For the full text of this licence, please go to: http://creativecommons.org/licenses/by-nc-nd/2.5/ 


\section{An asymmetric synthesis of trans-fused butyrolactones from endoperoxides}

Joshua Priest, Mark. R. Longland, Mark R. J. Elsegood and Marc C. Kimber*

Department of Chemistry, Loughborough University, Leicestershire, LE11 3TU, UK

M.C.Kimber@lboro.ac.uk.

\section{Abstract}

The intermolecular addition of 1,3-dicarbonyl equivalents to endoperoxides in the presence of an organocatalyst yields trans-fused butyrolactones in high yield and enantioselectivities. This methodology expands the synthetic utility of endoperoxides and further underlines their potential as sources of oxygen functionality for natural and non-natural product target synthesis.

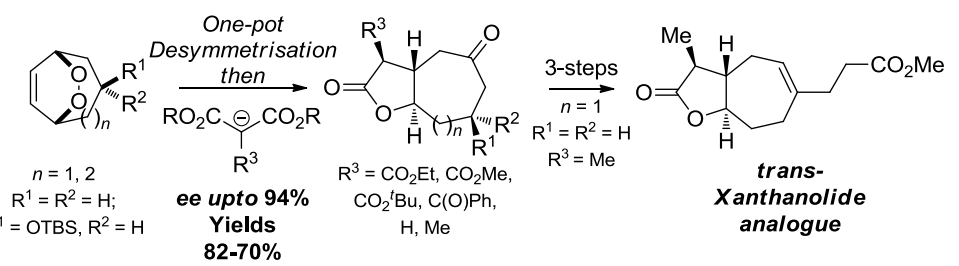


The oxidation of dienes to yield endoperoxides represents a selective and green chemical method for introducing oxygen functionality within a substrate. ${ }^{1}$ However, conversion of these endoperoxide products into useful asymmetric building blocks ${ }^{1 \mathrm{c}, 2}$ for natural and non-natural product target synthesis, without the use of toxic transition metals, has yet to be fully exploited. For example, Taylor and co-workers in 2002 reported the conversion of endoperoxides (1) into useful butyrolactones (2) via an intermediary cis-y-hydroxy-enone (3) (Scheme 1); however, they were only able to achieve this in an enantioselective fashion using a Co(II) catalyst and no asymmetric examples of bicyclic endoperoxides were included in their study, ${ }^{2 g}$ presumably due to the reactivity of bicyclic endoperoxides with $\mathrm{Co}(\mathrm{II})$ salts which traditionally delivers the bisepoxide products. ${ }^{3}$ In 2006, Toste and co-workers successfully desymmetrised bicyclic endoperoxides (4) using an organocatalytic KornblumDe La Mare rearrangement; ${ }^{4}$ however, the synthetic use of these highly enantio-enriched hydroxyenone products (5) has been limited. ${ }^{5}$

In a project aimed at investigating the anti-inflammatory activity of trans-fused xanthanolide natural product analogues, ${ }^{6}$ we recently required access to enantioenriched trans-fused butyrolactones (7) which we envisaged could be obtained from endoperoxides (6) (Scheme 1). While enantioselective routes toward cis-fused butyrolactones exist ${ }^{6 c-i}$ general synthetic routes toward trans-fused butyrolactones are less common; e.g. in Shishido's first asymmetric synthesis of the anti-inflammatory natural product xanthatin, ${ }^{6 j}$ they had to convert a key cis-lactone precursor $^{6 \mathrm{k}}$ into the trans-lactone using a 3-step procedure which included a Mitsunobu inversion of the crucial hydroxyl group.

Scheme 1. Planned route toward trans-fused butyrolactones.

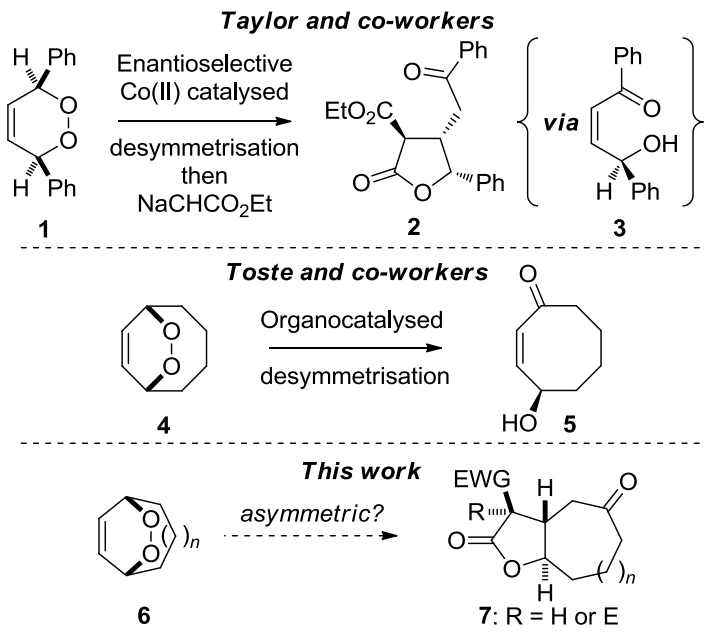

Key to this approach is the trapping of a cyclic hydroxyenone, a result of the based catalysed rearrangement of endoperoxides, by a 1,3dicarbonyl equivalent which we envisaged would occur via an intermolecular 1,4-conjugate addition pathway. ${ }^{2 g}$ Furthermore, since hydroxy enones such as 5 can be obtained via an organocatalysed Kornblum-De La Mare ${ }^{4,7}$ rearrangement of endoperoxides this approach would represent an asymmetric protocol for generating complex trans-fused butyrolactone scaffolds from endoperoxides, which in turn can be obtained from simple ${ }^{1} \mathrm{O}_{2}$ oxidation of dienes.

After initial optimisation studies ${ }^{9}$ we found treatment of endoperoxide $4^{8}$ in THF $^{9}$ in the presence of 10 mol\% of catalyst 8 a for a $16 \mathrm{~h}$ period followed by addition diethylsodiomalonate gave the desired lactone (-)-9a in an isolated yield of 76\% and ee of $92 \%$ (Scheme 1 and Table 1 ; entry 1). ${ }^{10,11}$ While the rearrangement of $\mathbf{4}$ with catalyst $\mathbf{8 b}$ with subsequent addition of diethylsodiomalonate gave lactone (+)-9a in a yield of $78 \%$ and ee of $94 \%$ (entry 2$)$.

Scheme 2. Lactone optimisation. 


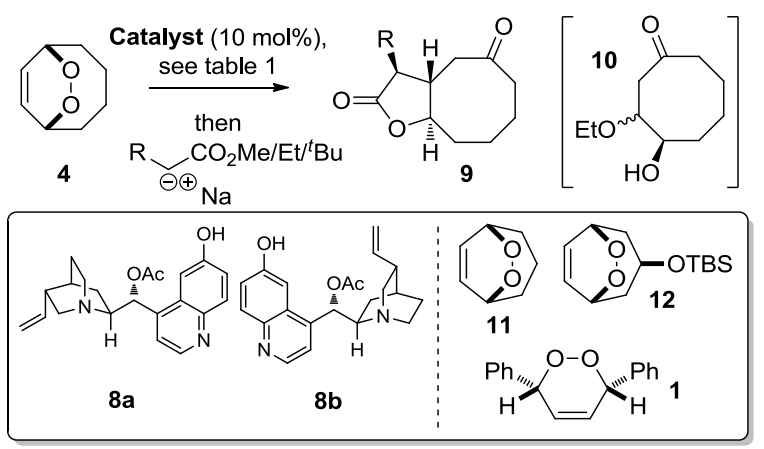

With optimal conditions for lactone formation determined we then looked at the scope of this sequence using other 1,3-dicarbonyls and endoperoxides in the presence of both catalysts $\mathbf{8 a}$ and $\mathbf{8 b}$ (Scheme 2 and Table 1). Undertaking the reaction with dimethylsodiomalonate and using catalyst $8 \mathbf{a}$ gave lactone (-)-9b in a comparable isolated yield and ee (entry 3 ). The use of ethyl sodioacetoacetate failed to deliver the butyrolactone but instead only yielded the alkoxy Michael addition product $\mathbf{1 0}$ as an inseparable mixture of diastereoisomers (entry 4 ); however, the addition of ethyl sodio benzoyl acetate gave the lactone (-)-9d using catalyst $8 \mathbf{a}$ in an isolated yield of $82 \%$ and an ee of $94 \%$ (entry 5). The endoperoxide 11, obtained in $87 \%$ yield from cycloheptadiene ${ }^{12}$ was also exposed to the optimised reaction conditions with catalyst 8 a delivering (-)-13 in $82 \%$ yield and ee of 78\%, while the antipode (+)-13 was obtained in an ee of $76 \%$ and a comparable isolated yield using catalyst $\mathbf{8 b}$ (entries 6 and 7, respectively). When the OTBS protected seven membered endoperoxide 12, derived from commercially available tropone,$^{13}$ was treated with catalyst $\mathbf{8 a}$ under the optimised conditions the lactone (-)-14 was isolated in a good yield of $74 \%$ and an excellent ee of $94 \%$, while catalyst $8 \mathrm{~b}$ gave the antipode (+)-14 in 70\% yield and an ee of $92 \%$ (entries 8 and 9 , respectively).

Table 1. Asymmetric butyrolactone formation and scope..$^{a, b}$

\begin{tabular}{|c|c|c|c|c|c|}
\hline Entry & $\begin{array}{c}\text { Endo- } \\
\text { peroxide }\end{array}$ & $\mathbf{R}$ & Product & $\begin{array}{l}\text { Yield } \\
{[\%]^{c}}\end{array}$ & $\mathrm{ee}^{d}$ \\
\hline 1 & 4 & $\mathrm{CO}_{2} \mathrm{Et}$ & $(-)-9 a$ & 76 & 92 \\
\hline $2^{e}$ & 4 & $\mathrm{CO}_{2} \mathrm{Et}$ & $(+)-9 a$ & 78 & 94 \\
\hline 3 & 4 & $\mathrm{CO}_{2} \mathrm{Me}$ & $(-)-9 b$ & 76 & 92 \\
\hline $4^{f}$ & 4 & $\mathrm{C}(\mathrm{O}) \mathrm{Me}$ & - & - & - \\
\hline 5 & 4 & $\mathrm{C}(\mathrm{O}) \mathrm{Ph}$ & $(-)-9 d$ & 82 & 94 \\
\hline 6 & 11 & $\mathrm{CO}_{2} \mathrm{Et}$ & $(-)-13$ & 82 & 78 \\
\hline $7^{e}$ & 11 & $\mathrm{CO}_{2} \mathrm{Et}$ & $(+)-13$ & 82 & 76 \\
\hline 8 & 12 & $\mathrm{CO}_{2} \mathrm{Et}$ & $(-)-14$ & 74 & 94 \\
\hline $9^{e}$ & 12 & $\mathrm{CO}_{2} \mathrm{Et}$ & $(+)-14$ & 70 & 92 \\
\hline 10 & 1 & $\mathrm{CO}_{2} \mathrm{Et}$ & $(+)-2$ & 44 & 10 \\
\hline
\end{tabular}

${ }^{\bar{a}}$ See the general experimental procedure. ${ }^{b} 10 \mathrm{~mol} \%$ of catalyst $8 \mathrm{a}$ used unless otherwise stated. ${ }^{c}$ Isolated yields. ${ }^{d}$ Determined by chiral HPLC. ${ }^{e} 10$ mol\% of catalyst $\mathbf{8 b}$. ${ }^{f} \mathbf{1 0}$ was isolated as an inseparable mixture of diastereoisomers. 
While the absolute stereochemistry of this later lactone can be deduced form the work of Toste ${ }^{4}$ the relative stereochemistry of $( \pm)-14$ was definitively assigned via X-ray crystallography. ${ }^{14}$ Importantly, the enantioselective synthesis of lactones containing a trans-fused 5,7-ring system gives us access to scaffolds ideal for investigating the biologically active xanthanolide class of natural products. ${ }^{6 a, b}$ Finally, endoperoxide 1 was exposed to the lactonisation conditions, but gave the known lactone (+)-2 in a disappointing ee of $10 \%$ (entry 10$)$.

Next we explored the installation of a methyl group at the alpha position of the trans-fused butyrolactone, as many natural product classes possess this substitution pattern (Scheme 3). Desymmetrisation of $\mathbf{4}$ using catalyst $\mathbf{8 b}$ followed by addition of diethylsodiomalonate gave the intermediate sodium salt 15 which upon treatment with iodomethane followed by acid mediated decarboxylation, gave the alpha methyl substituted lactone (+)-19 as the major diastereoisomer in $66 \%$ yield. ${ }^{15}$ Alternatively, the rearranged endoperoxide 4 could be treated with alpha-methyldiethylsodiomalonate giving lactone 17 which upon decarboxylation gave (+)-19 in a comparable chemical yield and diastereoselectivity. These two processes were also performed upon the seven membered endoperoxide $\mathbf{1 1}$ using catalyst $\mathbf{8 a}$ in this case, giving the desired alpha methyl substituted lactone (-)-20 in good chemical yield and diastereoselectivity. ${ }^{16}$

Scheme 3. Direct installation of an a-methyl group.

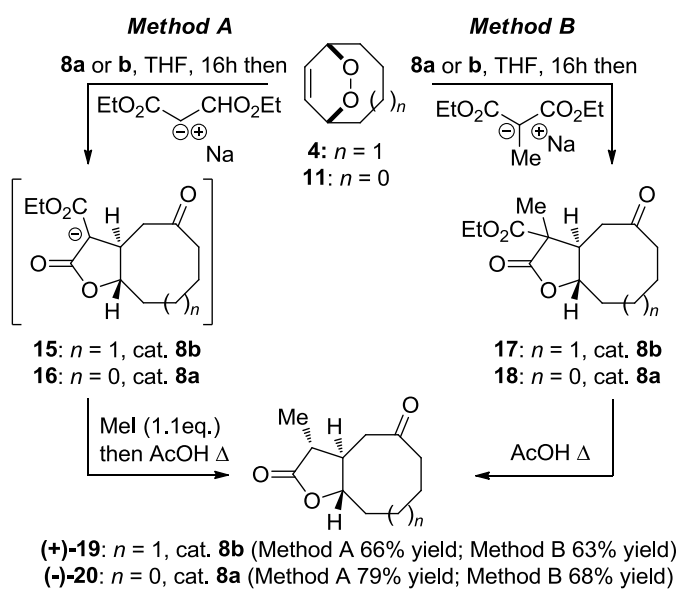

The trans-stereochemistry of the butyrolactone is installed via the mechanism shown below in Scheme 4 . The hydroxyenone $\mathbf{2 3}$ undergoes conjugate addition anti to the hydroxyl group, as depicted in $\mathbf{2 5}$, to deliver the addition product $\mathbf{2 4}$. This undergoes lactonisation to deliver the trans-fused product $\mathbf{2 6}$, which can then be protonated or trapped by an appropriate electrophile therefore giving $27 .{ }^{17}$

Scheme 4. Trans-fused lactone formation.

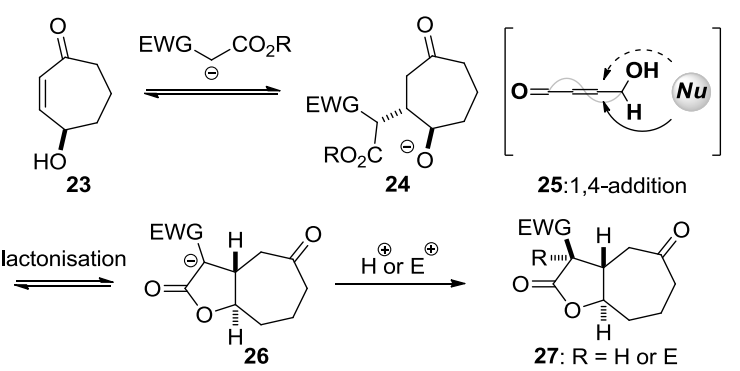

Finally, in reference to accessing xanthanolide analogues, we investigated the use of the ketone contained within the fused carbocycle as a synthetic handle (Scheme 5). Accordingly, we were able to selectively install an exo-methylene group using the conditions of Connell and coworkers ${ }^{18}$ on lactone (-)-20 giving 28 . This compound proved to be unstable and was directly reduced under Luche conditions ${ }^{19}$ to yield the allylic alcohol. Subsequent exposure of this alcohol to trimethyl orthoacetate, gave the Claisen rearrangement product (-)-29 in a yield $51 \%$ 
over 3-steps from (-)-20. Therefore using this approach we have a convenient asymmetric route to trans-fused xanthanolide analogues in just 5-steps and from readily available endoperoxides.

Scheme 5. Synthesis of xanthanolide analogue (-)-29.

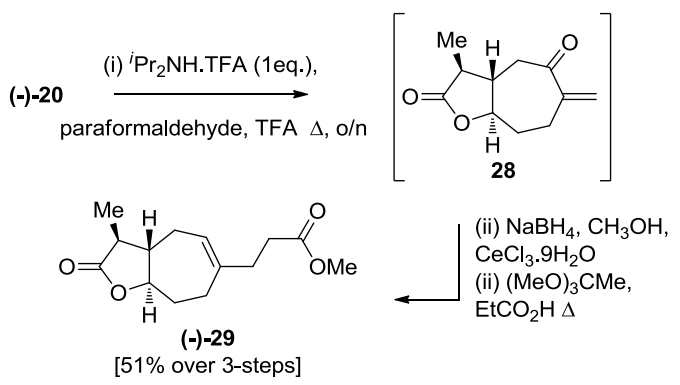

In summary, we have developed a convenient and single pot method for the synthesis of highly enantioenriched trans-fused butyrolactones from endoperoxides. Importantly, this process gives the desired products without the use of transitions metals and also showcases the use of endoperoxides as environmentally sustainable sources of oxygen functionality. The use of this methodology in the total synthesis of xanthanolide sesquiterpenoids and analogues for evaluation of their inflammatory activity is currently being pursued and will be reported on in due course.

\section{Experimental}

\section{General experimental procedure for enantio-enriched lactone formation.}

To a solution of the endoperoxide $(1 \mathrm{mmol})$ in dry THF $(5 \mathrm{~mL})$ was added the catalyst $(0.1 \mathrm{mmol}, \mathbf{8} \mathbf{a}$ or $\mathbf{8} \mathbf{b}$ as indicated below) and the resultant reaction mixture stirred at room temperature for $16 \mathrm{~h}$ under $\mathrm{N}_{2}$. After this period a solution of the desired nucleophile (1.0mmol) [prepared in THF $(3 \mathrm{~mL})$ by the addition of $\mathrm{NaOEt}$ or $\mathrm{NaOMe}(2.2 \mathrm{~mL}, 0.5 \mathrm{M}, 1.1 \mathrm{mmol})$ to the required malonate derivative $(1.0 \mathrm{mmol})]$ was added dropwise to the reaction mixture at $0^{\circ} \mathrm{C}$, and the resultant solution allowed to warm to room temperature and stirred for a further $16 \mathrm{~h}$ under $\mathrm{N}_{2}$. The reaction mixture was then cooled to $0^{\circ} \mathrm{C}$ and quenched by the addition of $1 \mathrm{M} \mathrm{HCl}$, after which it was partitioned between EtOAc $(50 \mathrm{~mL})$ and $\mathrm{H}_{2} \mathrm{O}(50 \mathrm{~mL})$, and the aqueous layer extracted with further portions of EtOAc $(2 \times 20 \mathrm{~mL})$. The organic layers were then combined, washed with brine, dried $\left(\mathrm{Na}_{2} \mathrm{SO}_{4}\right)$, filtered and the solvent removed in vacuo. The crude products were then purified by chromatography. Using this procedure then following compound were obtained:

$(-)-(3 R, 3 a S, 9 a R)$-Ethyl-2,5-dioxodecahydrocycloocta[b]furan-3-carboxylate ((-)-9a). Prepared using catalyst $8 a\left(193 \mathrm{mg}, 76 \% ; \mathrm{R}_{\mathrm{f}} 0.50 \mathrm{in}\right.$ 3:2 petroleum ether:ethyl acetate); $[\alpha]_{D}^{20}=-41.2$ (c 1.00, $\mathrm{CHCl}_{3}$ ); HRMS (ESI-orbitrap): $\mathrm{MNa}^{+}, \mathrm{C}_{12} \mathrm{H}_{16} \mathrm{O}_{5}$, found 277.1039, requires $\mathrm{MNa}^{+}$ 277.1052; IR (solution, $\left.\mathrm{CHCl}_{3}\right)$ 3032, 3019, 2942, 1784, 1734, 1708, 1209, $1159 \mathrm{~cm}^{-1} ;{ }^{1} \mathrm{H} \mathrm{NMR}\left(400 \mathrm{MHz} ; \mathrm{CDCl}_{3}\right) \delta 4.30(\mathrm{dq}, \mathrm{J}=2.4,7.2 \mathrm{~Hz}$, 2H), 4.19 (ddd, $J=3.2,8.8,10.0 \mathrm{~Hz}, 1 \mathrm{H}), 3.41(\mathrm{~d}, J=12.4 \mathrm{~Hz}, 1 \mathrm{H}), 3.19$ (ddt, $J=3.6,10.0,11.6 \mathrm{~Hz}, 1 \mathrm{H}), 2.78-2.68(\mathrm{~m}, 2 \mathrm{H}), 2.43(\mathrm{dd}, J=$ 11.6, $14.4 \mathrm{~Hz}, 1 \mathrm{H}), 2.35(\mathrm{dt}, J=5.6,12.8 \mathrm{~Hz}, 1 \mathrm{H}), 2.23-2.16(\mathrm{~m}, 1 \mathrm{H}), 1.90-1.70(\mathrm{~m}, 4 \mathrm{H}), 1.48-1.40(\mathrm{~m}, 1 \mathrm{H}), 1.31(\mathrm{t}, J=7.2 \mathrm{~Hz}, 3 \mathrm{H}) ;{ }^{13} \mathrm{C}$ NMR $\left(100 \mathrm{MHz} ; \mathrm{CDCl}_{3}\right) \delta 211.6,169.5,167.0,84.1,62.5,53.9,45.4,43.9,39.0,31.1,26.0,22.1,14.1 .3 S, 3 a R$, 9aS)-Ethyl-2,5dioxodecahydrocycloocta[b]furan-3-carboxylate ((+)-9a). Prepared using catalyst $8 \mathbf{b}(196 \mathrm{mg}, 78 \%) ;[\alpha]_{\mathrm{D}}{ }^{18}=+45.2\left(\mathrm{c} 1.00, \mathrm{CHCl}_{3}\right)$.

(-)-(3R, 3aS, 9aR)-Methyl-2,5-dioxodecahydrocycloocta[b]furan-3-carboxylate ((-)-9b). Prepared using catalyst $8 \mathrm{a}\left(182 \mathrm{mg}, 76 \% ; \mathrm{R}_{\mathrm{f}} 0.20\right.$ in 1:1 petroleum ether/ethyl acetate; MP $70.0-71.5 \stackrel{\circ}{\circ}$ ); $[\alpha]_{D}^{20}=-58.0\left(c 1.00, \mathrm{CHCl}_{3}\right)$; HRMS (ESI-orbitrap): $\mathrm{MNa}^{+}, \mathrm{C}_{12} \mathrm{H}_{16} \mathrm{O}_{5}$, found 263.0883, requires $\mathrm{MNa}^{+}$263.0895; IR (solution, $\mathrm{CHCl}_{3}$ ) 3033, 3019, 2946, 1785, 1743, 1708, 1216, $1209,1159 \mathrm{~cm}^{-1}$; ${ }^{1} \mathrm{H} \mathrm{NMR}(400 \mathrm{MHz}$; $\left.\mathrm{CDCl}_{3}\right) \delta 4.18(\mathrm{ddd}, J=3.2,8.8,11.2 \mathrm{~Hz}, 1 \mathrm{H}), 3.86(\mathrm{~s}, 3 \mathrm{H}), 3.42(\mathrm{~d}, J=12.0 \mathrm{~Hz}, 1 \mathrm{H}), 3.25-3.18(\mathrm{~m}, 1 \mathrm{H}), 2.80-2.71(\mathrm{~m}, 1 \mathrm{H}), 2.75(\mathrm{dd}, J=$ 
4.0, $14.8 \mathrm{~Hz}, 1 \mathrm{H}), 2.43(\mathrm{dd}, J=11.6,14.4 \mathrm{~Hz}, 1 \mathrm{H}), 2.36$ (dt, J = 5.2, $12.8 \mathrm{~Hz}, 1 \mathrm{H}), 2.24-2.16(\mathrm{~m}, 1 \mathrm{H}), 1.95-1.72(\mathrm{~m}, 4 \mathrm{H}), 1.50-1.41(\mathrm{~m}$, $1 \mathrm{H}) ;{ }^{13} \mathrm{C} \mathrm{NMR}\left(100 \mathrm{MHz} ; \mathrm{CDCl}_{3}\right) \delta 211.4,169.3,167.0,84.1,53.8,53.3,45.5,43.9,39.0,31.1,26.0,22.1$.

(-)-(3S,3aS,9aR)-tert-Butyl 2,5-dioxodecahydrocycloocta[b]furan-3-carboxylate ((-)-9c). Prepared using catalyst 8a (191mg, $68 \% ; R_{f} 0.65$ in 1:1 petroleum ether/ethyl acetate; MP $\left.87-89{ }^{\circ} \mathrm{C}\right) ;[\alpha]_{D}^{26}=-29.6\left(c 1.00, \mathrm{CHCl}_{3}\right) ; \mathrm{HRMS}^{2}$ ESI-orbitrap): $\mathrm{MNa}^{+}, \mathrm{C}_{15} \mathrm{H}_{22} \mathrm{O}_{5}$, found 305.1353 , requires $\mathrm{MNa}^{+}$305.1365; IR (solution, $\mathrm{CHCl}_{3}$ ) 2938, 1783, 1727, 1709, 1459, 1371, $1221 \mathrm{~cm}^{-1} ;{ }^{1} \mathrm{H} \mathrm{NMR}\left(400 \mathrm{MHz} ; \mathrm{CDCl}_{3}\right) \delta 4.11(\mathrm{ddd}, \mathrm{J}=$ 3.2, 8.4, $11.6 \mathrm{~Hz}, 1 \mathrm{H}), 3.26(\mathrm{~d}, J=12.4 \mathrm{~Hz}, 1 \mathrm{H}), 3.12$ (ddt, J=3.6, 10.0, $14.0 \mathrm{~Hz}, 1 \mathrm{H}), 2.77-2.70(\mathrm{~m}, 1 \mathrm{H}), 2.68(\mathrm{dd}, J=4.0,14.8 \mathrm{~Hz}, 1 \mathrm{H})$, $2.41(\mathrm{dd}, J=12.0,14.8 \mathrm{~Hz}, 1 \mathrm{H}), 2.33(\mathrm{dt}, J=5.2,12.8 \mathrm{~Hz}, 1 \mathrm{H}), 2.19-2.12(\mathrm{~m}, 1 \mathrm{H}), 1.90-1.69(\mathrm{~m}, 5 \mathrm{H}), 1.49(\mathrm{~s}, 9 \mathrm{H}) ;{ }^{13} \mathrm{C} \mathrm{NMR}(100 \mathrm{MHz}$; $\left.\mathrm{CDCl}_{3}\right) \delta 211.8,169.8,165.7,83.8,83.4,54.6,45.5,43.7,39.0,31.0,28.0,26.1,22.0$.

(-)-(3R,3aS,9aR)-3-Benzoylhexahydrocycloocta[b]furan-2,5(3H,6H)-dione ((-)-9d). Prepared using catalyst $8 \mathrm{a}\left(234 \mathrm{mg}, 82 \%\right.$; $\mathrm{R}_{\mathrm{f}} 0.55 \mathrm{in}$ 1:1 petroleum ether/ethyl acetate; MP $103-105 \stackrel{\circ}{\circ}$ ); $[\alpha]_{D}^{26}=-122.8$ (c 1.00, $\mathrm{CHCl}_{3}$ ); HRMS (ESI-orbitrap): $\mathrm{MNa}^{+}, \mathrm{C}_{17} \mathrm{H}_{18} \mathrm{O}_{4}$, found 309.1091 , requires $\mathrm{MNa}^{+}$309.1103; IR (solution, $\mathrm{CHCl}_{3}$ ) 2939, 1773, 1706, 1685, 1449, $1270 \mathrm{~cm}^{-1} ;{ }^{1} \mathrm{H} \mathrm{NMR}\left(400 \mathrm{MHz} ; \mathrm{CDCl}_{3}\right) \delta 8.05-8.03(\mathrm{~m}, 2 \mathrm{H})$, $7.67-7.63(\mathrm{~m}, 1 \mathrm{H}), 7.55-7.51(\mathrm{~m}, 2 \mathrm{H}), 4.38(\mathrm{~d}, J=12.0 \mathrm{~Hz}, 1 \mathrm{H}), 4.27(\mathrm{ddd}, J=3.6,8.4,10.0 \mathrm{~Hz}, 1 \mathrm{H}), 3.71-3.62(\mathrm{~m}, 1 \mathrm{H}), 2.94-2.88(\mathrm{~m}$ $1 \mathrm{H}), 2.68(\mathrm{dd}, J=4.0,14.8 \mathrm{~Hz}, 1 \mathrm{H}), 2.39-2.33(\mathrm{~m}, 1 \mathrm{H}), 2.23-2.19(\mathrm{~m}, 1 \mathrm{H}), 1.96-1.84(\mathrm{~m}, 4 \mathrm{H}), 1.52-1.48(\mathrm{~m}, 1 \mathrm{H}) ;{ }^{13} \mathrm{C} \mathrm{NMR}(100 \mathrm{MHz}$; $\left.\mathrm{CDCl}_{3}\right) \delta 211.9,191.7,170.0,135.8,134.4,129.6,128.9,84.1,55.5,45.8,41.9,38.6,31.2,26.3,21.7$.

(-)-((3R, 3aS, 8aR)-ethyl 2,5-dioxooctahydro-2H-cyclohepta[b]furan-3-carboxylate ((-)-13). Prepared using catalyst 8a (193mg, 82\%; $\mathrm{R}_{f}$

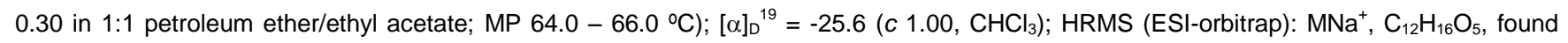
263.1130, requires $\mathrm{MNa}^{+}$263.0895; IR (solution, $\mathrm{CHCl}_{3}$ ) 3029, 2957, 2931, 2859, 1789, 1736, 1709, $1016 \mathrm{~cm}^{-1} ;{ }^{1} \mathrm{H} \mathrm{NMR}\left(400 \mathrm{MHz} ; \mathrm{CDCl}_{3}\right) \delta$ 4.26 (dq, $J=1.6,7.2 \mathrm{~Hz}, 2 \mathrm{H}), 4.09-4.01(\mathrm{~m}, 1 \mathrm{H}), 3.34$ (d, $J=12.4 \mathrm{~Hz}, 1 \mathrm{H}), 3.04$ (ddt, $J=4.4,10.4,12.0 \mathrm{~Hz}, 1 \mathrm{H}), 2.78(\mathrm{dd}, J=4.0,18.0 \mathrm{~Hz}$, $1 \mathrm{H}), 2.63(\mathrm{dt}, J=2.4,13.2 \mathrm{~Hz}, 1 \mathrm{H}), 2.58-2.52(\mathrm{~m}, 1 \mathrm{H}), 2.50-2.43(\mathrm{~m}, 1 \mathrm{H}), 2.37(\mathrm{dd}, J=12.0,18.0 \mathrm{~Hz}, 1 \mathrm{H}), 2.11-2.02(\mathrm{~m}, 1 \mathrm{H}), 1.80-$ $1.71(\mathrm{~m}, 1 \mathrm{H}), 1.58-1.48(\mathrm{~m}, 1 \mathrm{H}), 1.28(\mathrm{t}, J=7.6 \mathrm{~Hz}, 3 \mathrm{H}) ;{ }^{13} \mathrm{C} \mathrm{NMR}\left(100 \mathrm{MHz} ; \mathrm{CDCl}_{3}\right) \delta 209.4,169.9,166.7,83.6,62.5,53.1,43.3,43.1$, 42.1, 34.0, 20.7, 14.1. (+)-((3S, 3aR, 8aS)-ethyl 2,5-dioxooctahydro-2H-cyclohepta[b]furan-3-carboxylate ((+)-13). Prepared using catalyst $8 \mathbf{b}(188 \mathrm{mg}, 81 \%) ;[\alpha]_{\mathrm{D}}^{21}=+29.6\left(\right.$ c $\left.1.00, \mathrm{CHCl}_{3}\right)$.

(-)-(3R, 3aS, 7S, 8aR)-Ethyl 7-(tert-butyldimethylsilyloxy)-2,5-dioxooctahydro-2H-cyclohepta[b]furan-3-carboxylate ((-)-14). Using the general method but on a $0.40 \mathrm{mmol}$ scale and prepared using catalyst $8 \mathrm{a}$ (107mg, 74\%; $\mathrm{R}_{\mathrm{f}} 0.45$ in $3: 1$ petroleum ether/ethyl acetate; MP $102.0-103.5 \stackrel{\circ}{\circ}) ;[\alpha]_{D}^{19}=-35.8\left(c\right.$ 1.00, $\left.\mathrm{CHCl}_{3}\right) ; \mathrm{HRMS}$ (ESI-orbitrap): $\mathrm{MNa}^{+}, \mathrm{C}_{18} \mathrm{H}_{30} \mathrm{O}_{6} \mathrm{Si}$, found 393.1696, requires $\mathrm{MNa}^{+} 393.1709 ; \mathrm{IR}$ (solution, $\mathrm{CHCl}_{3}$ ) 2957, 2931, 2858, 1789, 1736, 1708, 1260, $1016 \mathrm{~cm}^{-1} ;{ }^{1} \mathrm{H} \mathrm{NMR}\left(400 \mathrm{MHz} ; \mathrm{CDCl}_{3}\right) \delta 4.28$ (dq, J=2.8, $\left.7.2 \mathrm{~Hz}, 2 \mathrm{H}\right), 4.10$ (ddd, $J=3.6,10.0,11.6 \mathrm{~Hz}, 1 \mathrm{H}), 3.93-3.87(\mathrm{~m}, 1 \mathrm{H}), 3.30(\mathrm{~d}, J=13.2 \mathrm{~Hz}, 1 \mathrm{H}), 3.30-3.19(\mathrm{~m}, 1 \mathrm{H}), 3.04(\mathrm{dd}, J=11.2,12.0 \mathrm{~Hz}, 1 \mathrm{H}), 2.87$ (dd, $J=4.8,17.6 \mathrm{~Hz}, 1 \mathrm{H}), 2.68-2.64(\mathrm{~m}, 2 \mathrm{H}), 2.29(\mathrm{dd}, J=11.6,17.6 \mathrm{~Hz}, 1 \mathrm{H}), 2.03-1.95(\mathrm{~m}, 1 \mathrm{H}), 1.32(\mathrm{t}, J=7.2 \mathrm{~Hz}, 3 \mathrm{H}), 0.87(\mathrm{~s}, 9 \mathrm{H})$, 0.08 (s, 6H); ${ }^{13} \mathrm{C}$ NMR (100 MHz; $\left.\mathrm{CDCl}_{3}\right) \delta$ 205.7, 169.4, 166.4, 79.0, 66.1, 62.6, 53.3, 44.8, 43.8, 41.4, 31.0, 25.6, 18.0, 14.1, -4.9. (+)-(3S, $3 a R, 7 R, 8 a S)$-Ethyl 7-(tert-butyldimethylsilyloxy)-2,5-dioxooctahydro-2H-cyclohepta[b]furan-3-carboxylate ((+)-14). Using the general method and prepared using catalyst $8 \mathrm{~b}(102 \mathrm{mg}, 70 \%) ;[\alpha]_{D}{ }^{18}=+32.6\left(c 1.00, \mathrm{CHCl}_{3}\right)$.

General methylation conditions. Method A: To a solution of the endoperoxide 4 or $11(1.00 \mathrm{mmol})$ in dry THF (10mL) was added the catalyst $8 \mathbf{a}$ or $\mathbf{8 b}(0.1 \mathrm{mmol})$ and the resultant reaction mixture stirred at room temperature for $16 \mathrm{~h}$ under $\mathrm{N}_{2}$. After this period a solution of $\mathrm{NaCH}\left(\mathrm{CO}_{2} \mathrm{Et}\right)_{2}$ [1.1mmol; prepared from $\mathrm{H}_{2} \mathrm{C}\left(\mathrm{CO}_{2} \mathrm{Et}\right)_{2}(193 \mathrm{mg}, 1.10 \mathrm{mmol})$ and $\mathrm{NaOEt}(2.30 \mathrm{~mL}, 0.5 \mathrm{M}$ soln., $\left.1.15 \mathrm{mmol}) \mathrm{THF}(5 \mathrm{~mL})\right]$ was added dropwise to the reaction mixture at $0^{\circ} \mathrm{C}$, and the resultant solution allowed to warm to room temperature and stirred for a further $16 \mathrm{~h}$ under $\mathrm{N}_{2}$. 
The reaction mixture was then cooled to $0^{\circ} \mathrm{C}$ and iodomethane $(94 \mu \mathrm{L}, 1.5 \mathrm{mmol})$ was added after which the reaction mixture was stirred for a further $16 \mathrm{~h}$ at room temperature. A sat. solution of $\mathrm{NH}_{4} \mathrm{Cl}(10 \mathrm{~mL})$ was then added and the solution then partitioned between $\mathrm{CH}_{2} \mathrm{Cl}_{2}(50 \mathrm{~mL})$ and $\mathrm{H}_{2} \mathrm{O}(50 \mathrm{~mL})$, and the aqueous layer extracted with further portions of $\mathrm{CH}_{2} \mathrm{Cl}_{2}(2 \times 40 \mathrm{~mL})$. The organic layers were then combined, washed with brine $(50 \mathrm{~mL})$, dried $\left(\mathrm{Na}_{2} \mathrm{SO}_{4}\right)$, filtered and the solvent removed in vacuo. The crude material was then dissolved in $50 \% \mathrm{AcOH}(10 \mathrm{~mL})$ and refluxed overnight. After this period, the reaction was cooled, carefully basified with $\mathrm{NaHCO}_{3}$ and the aqueous layer extracted with dichloromethane $(2 \times 40 \mathrm{~mL})$. The combined organic extracts were then dried $\left(\mathrm{Na}_{2} \mathrm{SO}_{4}\right)$, filtered, and the solvent was removed in vacuo. The crude product was then purified by column chromatography. Method B: To a solution of the endoperoxide 4 or 11 (1.0mmol) in dry THF $(8 \mathrm{~mL})$ was added the catalyst $\mathbf{8 a}$ or $\mathbf{8 b}(35 \mathrm{mg}, 0.1 \mathrm{mmol})$ and the resultant reaction mixture stirred at room temperature for $16 \mathrm{~h}$ under $\mathrm{N}_{2}$. After this period a solution of $\mathrm{NaCMe}\left(\mathrm{CO}_{2} \mathrm{Et}\right)_{2}$ [1.1mmol; prepared from $\mathrm{HCMe}\left(\mathrm{CO}_{2} \mathrm{Et}\right)_{2}(193 \mathrm{mg}, 1.10 \mathrm{mmol})$ and $\mathrm{NaOEt}(2.30 \mathrm{~mL}, 0.5 \mathrm{M}$ soln., $1.15 \mathrm{mmol})$ THF $(5 \mathrm{~mL})]$ was added dropwise to the reaction mixture at $0^{\circ} \mathrm{C}$, and the resultant solution allowed to warm to room temperature and stirred for a further $16 \mathrm{~h}$ under $\mathrm{N}_{2}$. The reaction mixture was then cooled to $0^{\circ} \mathrm{C}$ and a sat. soln. of $\mathrm{NH}_{4} \mathrm{Cl}(10 \mathrm{~mL})$ added, partitioned between $\mathrm{CH}_{2} \mathrm{Cl}_{2}(50 \mathrm{~mL})$ and $\mathrm{H}_{2} \mathrm{O}(50 \mathrm{~mL})$, and the aqueous layer extracted with further portions of $\mathrm{CH}_{2} \mathrm{Cl}_{2}(2 \times 30 \mathrm{~mL})$. The organic layers were then combined, washed with brine $(50 \mathrm{~mL})$, dried $\left(\mathrm{Na}_{2} \mathrm{SO}_{4}\right)$, filtered and the solvent removed in vacuo. The crude material was then dissolved in $50 \% \mathrm{AcOH}(10 \mathrm{~mL})$ and refluxed overnight. After this period, the reaction was cooled, carefully basified with $\mathrm{NaHCO}_{3}$ and the aqueous layer extracted with $\mathrm{CH}_{2} \mathrm{Cl}_{2}(2 \times 40 \mathrm{~mL})$. The combined organic extracts were then dried $\left(\mathrm{Na}_{2} \mathrm{SO}_{4}\right)$, filtered, and the solvent was removed in vacuo. The crude product was then purified by column chromatography. Using these methods the following compounds were obtained.

(+)-(3R, 3aR, 9aS)-3-Methylhexahydrocycloocta[b]furan-2,5(3H,6H)-dione ((+)-19). Obtained as a colourless oil (Method A 130mg, 66\%;

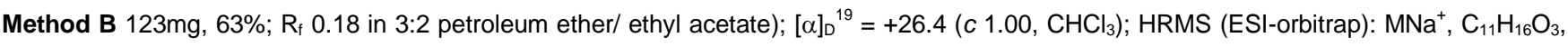
found 219.0992, requires $\mathrm{MNa}^{+}$219.0997; IR (solution, $\left.\mathrm{CHCl}_{3}\right)$ 3025, 2938, 1774, 1705, $991 \mathrm{~cm}^{-1} ;{ }^{1} \mathrm{H} \mathrm{NMR}(400 \mathrm{MHz} ; \mathrm{CDCl}$ ) $\delta 4.11(\mathrm{dt}, \mathrm{J}=$ 3.2, $9.2 \mathrm{~Hz}, 1 \mathrm{H}), 2.66(\mathrm{dd}, 3.6,10.4 \mathrm{~Hz}, 1 \mathrm{H}), 2.68-2.61(\mathrm{~m}, 1 \mathrm{H}), 2.45(\mathrm{dd}, J=11.6,14.0 \mathrm{~Hz}, 1 \mathrm{H}), 2.40-2.35(\mathrm{~m}, 1 \mathrm{H}), 2.34(\mathrm{q}, J=6.8 \mathrm{~Hz}$, $1 \mathrm{H}), 2.27-2.19(\mathrm{~m}, 2 \mathrm{H}), 1.95-1.85(\mathrm{~m}, 1 \mathrm{H}), 1.85-1.72(\mathrm{~m}, 2 \mathrm{H}), 1.68-1.59(\mathrm{~m}, 1 \mathrm{H}), 1.54-1.46(\mathrm{~m}, 1 \mathrm{H}), 1.26(\mathrm{~d}, \mathrm{~J}=6.8 \mathrm{~Hz}, 3 \mathrm{H}) ;{ }^{13} \mathrm{C}$ $\mathrm{NMR}\left(100 \mathrm{MHz} ; \mathrm{CDCl}_{3}\right) \delta 212.8,177.1,84.0,49.0,45.3,42.6,39.9,31.4,25.8,22.9,12.8$.

(-)-(3S, 3aS, 8aR)-3-Methylhexahydro-2H-cyclohepta[b]furan-2,5(3H)-dione ((-)-20). Obtained as a waxy solid (Method A (performed on a 2mmol scale) 298mg, 79\%; Method B 124mg, 68\%; $\mathrm{R}_{\mathrm{f}} 0.35$ in 1:1 petroleum ether/ethyl acetate; MP $\left.28-31^{\circ} \mathrm{C}\right) ;[\alpha]_{\triangleright}{ }^{19}=-92\left(c 1.00, \mathrm{CHCl}_{3}\right)$; HRMS (ESI-orbitrap): $\mathrm{MNa}^{+}, \mathrm{C}_{10} \mathrm{H}_{14} \mathrm{O}_{3}$, found 205.0836, requires $\mathrm{MNa}^{+} 205.0841$; IR (solution, $\mathrm{CHCl}_{3}$ ) 3029, 3019, 2936, 1776, 1703, 1208 $\mathrm{cm}^{-1} ;{ }^{1} \mathrm{H}$ NMR $\left(400 \mathrm{MHz} ; \mathrm{CDCl}_{3}\right.$ ) $\delta 3.97$ (ddd, $\left.J=3.2,9.6,10.8 \mathrm{~Hz}, 1 \mathrm{H}\right), 2.78$ (dd, $\left.J=4.0,18.0 \mathrm{~Hz}, 1 \mathrm{H}\right), 2.51-2.47(\mathrm{~m}, 2 \mathrm{H}), 2.36-2.28(\mathrm{~m}$, $1 \mathrm{H}), 2.20-2.15(\mathrm{~m}, 2 \mathrm{H}), 2.12-2.08(\mathrm{~m}, 1 \mathrm{H}), 2.07-2.03(\mathrm{~m}, 1 \mathrm{H}), 1.72-1.53(\mathrm{~m}, 2 \mathrm{H}), 1.26(\mathrm{~d}, J=7.6 \mathrm{~Hz}, 3 \mathrm{H}) ;{ }^{13} \mathrm{C} \mathrm{NMR}(100 \mathrm{MHz} ; \mathrm{CDCl} 3) \delta$ 210.3, 177.4, 83.6, 46.2, 43.5, 43.4, 41.9, 34.3, 20.8, 12.9.

(+)-(3aR, 9aS)-Hexahydrocycloocta[b]furan-2,5(3H,6H)-dione ((+)-21). Lactone (+)-9a (0.36g, 1.4mmol) was dissolved ethanol (15mL) and $2 \mathrm{M} \mathrm{KOH}(15 \mathrm{~mL})$ and the resultant solution stirred at room temperature for $16 \mathrm{~h}$. After this period the reaction mixture was carefully acidified with $5 \mathrm{M} \mathrm{HCl}$, extracted with $\mathrm{CH}_{2} \mathrm{Cl}_{2}(2 \times 30 \mathrm{~mL})$, and the combined organic layers dried $\left(\mathrm{Na}_{2} \mathrm{SO}_{4}\right)$, filtered and the solvent removed in vacuo. The crude acid was then dissolved in toluene $(25 \mathrm{~mL})$ and refluxed for overnight. The solvent was then removed in vacuo and the crude solid purified by column chromatography $\left(\mathrm{R}_{f} 0.13,3: 2\right.$ petroleum ether/ethyl acetate) to yield the title compound as a colourless solid (194mg, $76 \%$; MP $74-76 \stackrel{\circ}{\circ}) ;[\alpha]_{D}^{19}=+21.2\left(c 1.00, \mathrm{CHCl}_{3}\right)$; HRMS (ESI-orbitrap): $\mathrm{MNa}^{+}, \mathrm{C}_{10} \mathrm{H}_{14} \mathrm{O}_{3}$, found 205.0836, requires $\mathrm{MNa}^{+} 205.0841 ; \mathrm{IR}$ (solution, $\left.\mathrm{CHCl}_{3}\right) 3011,3027,2942,1780,1705,1016 \mathrm{~cm}^{-1} ;{ }^{1} \mathrm{H}$ NMR $\left(400 \mathrm{MHz} ; \mathrm{CDCl}_{3}\right) \delta 4.22(\mathrm{dt}, J=3.2,9.2 \mathrm{~Hz}, 1 \mathrm{H}), 2.77-2.64(\mathrm{~m}, 4 \mathrm{H}), 2.48(\mathrm{dd}, J$ $=11.2,13.6 \mathrm{~Hz}, 1 \mathrm{H}), 2.42-2.34(\mathrm{~m}, 2 \mathrm{H}), 2.27-2.21(\mathrm{~m}, 1 \mathrm{H}), 1.93-1.76(\mathrm{~m}, 3 \mathrm{H}), 1.72-1.63(\mathrm{~m}, 1 \mathrm{H}), 1.54-1.46(\mathrm{~m}, 1 \mathrm{H}) ;{ }^{13} \mathrm{C} \mathrm{NMR}(100$ $\left.\mathrm{MHz} ; \mathrm{CDCl}_{3}\right) \delta 21.4,174.4,86.0,46.4,41.1,39.7,37.1,31.6,25.7,22.8$. 
(-)-(3aS ,8aR)-hexahydro-2H-cyclohepta[b]furan-2,5(3H)-dione ((-)-22). Lactone (-)-13 (241mg, 1.00mmol) was dissolved in 50\% AcOH $(10 \mathrm{~mL})$ and refluxed overnight. After this period, the reaction was cooled, basified with $\mathrm{NaHCO}_{3}$ and the aqueous layer extracted with dichloromethane $(2 \times 30 \mathrm{~mL})$. The combined organic extracts were then dried $\left(\mathrm{Na}_{2} \mathrm{SO}_{4}\right)$, filtered, and the solvent was removed in vacuo. The crude product were then purified by chromatography giving the title compound as a colourless solid $(130 \mathrm{mg}, 78 \% ; \mathrm{MP} 69.0-70.5 \stackrel{\circ}{\circ} \mathrm{C}) ;[\alpha]_{\mathrm{D}}^{19}=$ -59.6 (c 1.00, $\mathrm{CHCl}_{3}$ ); HRMS (ESI-orbitrap): $\mathrm{MNa}^{+}, \mathrm{C}_{9} \mathrm{H}_{12} \mathrm{O}_{3}$, found 191.0679, requires $\mathrm{MNa}^{+}$191.0684; IR (solution, $\mathrm{CHCl}_{3}$ ) 3019, 2950, 1782, 1704, $1211 \mathrm{~cm}^{-1} ;{ }^{1} \mathrm{H}$ NMR (400 MHz; $\mathrm{CDCl}_{3}$ ) $\delta 4.03$ (ddd, $\left.J=3.6,5.2,9.6 \mathrm{~Hz}, 1 \mathrm{H}\right), 2.80$ (dd, J = 3.6, $\left.18.0 \mathrm{~Hz}, 1 \mathrm{H}\right), 2.74-2.52(\mathrm{~m}, 4 \mathrm{H}), 2.48$ (dq, $J=3.6,12.8 \mathrm{~Hz}, 1 \mathrm{H}), 2.40-2.30(\mathrm{~m}, 2 \mathrm{H}), 2.12-2.04(\mathrm{~m}, 1 \mathrm{H}), 1.72(\mathrm{ddt}, J=4.0,11.2,13.2 \mathrm{~Hz}, 1 \mathrm{H}), 1.61-1.50(\mathrm{~m}, 1 \mathrm{H}) ;{ }^{13} \mathrm{C} \mathrm{NMR}(100$ $\left.\mathrm{MHz} ; \mathrm{CDCl}_{3}\right) \delta 210.3,175.0,85.6,44.1,43.3,38.6,35.9,34.2,20.8$.

(-)-Methyl 3-((3S, 3aS, 8aR)-3-methyl-2-oxo-3, 3a, 4, 7, 8, 8a-hexahydro-2H-cyclohepta[b]furan-6-yl)propanoate ((-)-29). To a mixture of (-)-20 (282mg, 1.55mmol) and paraformaldehyde (94mg, 3.10mmol) in dry THF (2mL) was added ('Pr) ${ }_{2} \mathrm{NH}$. TFA (334mg, $\left.1.55 \mathrm{mmol}\right)$ and TFA $(12 \mu \mathrm{L}, 0.16 \mathrm{mmol})$. The reaction mixture was then refluxed for $2 \mathrm{~h}$, after which it was cooled to room temperature and another portion of paraformaldehyde $(94 \mathrm{mg}, 3.10 \mathrm{mmol})$ added and the reaction mixture refluxed for a further $6 \mathrm{~h}$. After this period the reaction mixture was cooled, the solvent removed in vacuo and the residue dissolved in $\mathrm{CH}_{2} \mathrm{Cl}_{2}(40 \mathrm{~mL})$ and washed sequentially with $1 \mathrm{M} \mathrm{HCl}(30 \mathrm{~mL}), \mathrm{NaHCO}$ $(30 \mathrm{~mL})$ and finally brine $(30 \mathrm{~mL})$. The combined organic layers were then dried $\left(\mathrm{Na}_{2} \mathrm{SO}_{4}\right)$, filtered and the solvent removed in vacuo. The crude oil was then purified by chromatography $\left(R_{f} 0.4\right.$ in 3:1 ethyl acetate/petroleum ether) to give the desired methenylated compound as a colourless oil (182mg, 61\%) which was used without further purification. The methenylated compound (160mg, 0.825mmol) was dissolved in methanol $(6 \mathrm{~mL})$ and to this solution was added $\mathrm{CeCl}_{3} .9 \mathrm{H}_{2} \mathrm{O}(308 \mathrm{mg}, 0.825 \mathrm{mmol})$. The reaction mixture was cooled to $0{ }^{\circ} \mathrm{C}$ and to the stirring solution was added $\mathrm{NaBH}_{4}(102 \mathrm{mg}, 2.68 \mathrm{mmol})$ in portions over $20 \mathrm{~min}$. After the addition the reaction mixture was then monitered by tlc until the disapperance of the starting material was detected. After approx. $1 \mathrm{~h}$ the reaction was cooled once again cooled to $0^{\circ} \mathrm{C}$ and carefully quenched with a sat. solution of $\mathrm{NH}_{4} \mathrm{Cl}$. The reaction mixture was then transferred to a separating funnel and the aqueous extracted with ethyl acetate $(3 \times 20 \mathrm{~mL})$, and the resultant combined organic extracts dried $\left(\mathrm{Na}_{2} \mathrm{SO}_{4}\right)$, filterred and the solvent finally removed in vacuo. The crude product was then immediately dissolved in trimethyl orthoacetate $(1.00 \mathrm{~mL})$ and to this mixture was added a propionic acid (1drop). This solution was then heated for $16 \mathrm{~h}$ at reflux under a $\mathrm{N}_{2}$ atmosphere. After this period the reaction mixture was cooled and the residual trimethyl orthoacetate removed in vacuo and the crude product purified by column chromatography $\left(R_{f} 0.55\right.$ in $5: 3$ ethyl acetate/petroleum ether) giving

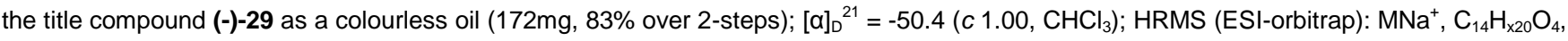
found 275.1256, requires $\mathrm{MNa}^{+}$275.1259; IR (solution, $\left.\mathrm{CHCl}_{3}\right)$ 2900, 1765, 1733, $1440 \mathrm{~cm}^{-1} ;{ }^{1} \mathrm{H} \mathrm{NMR}\left(400 \mathrm{MHz} ; \mathrm{CDCl}_{3}\right) \delta 5.77-5.73(\mathrm{~m}$, $1 \mathrm{H}), 3.83(\mathrm{ddd}, J=4.0,10.0,11.2 \mathrm{~Hz}, 1 \mathrm{H}), 3.67(\mathrm{~s}, 3 \mathrm{H}), 2.91-2.86(\mathrm{~m}, 1 \mathrm{H}), 2.79(\mathrm{q}, J=6.8 \mathrm{~Hz}, 1 \mathrm{H}), 2.52-2.41(\mathrm{~m}, 4 \mathrm{H}), 2.39-2.35(\mathrm{~m}$, $2 \mathrm{H}), 2.24-2.16(\mathrm{~m}, 1 \mathrm{H}), 2.11-2.01(\mathrm{~m}, 1 \mathrm{H}), 1.81-1.75(\mathrm{~m}, 1 \mathrm{H}), 1.71-1.60(\mathrm{~m}, 1 \mathrm{H}), 1.31(\mathrm{~d}, J=6.8 \mathrm{~Hz}, 3 \mathrm{H}) ;{ }^{13} \mathrm{C} \mathrm{NMR}(100 \mathrm{MHz} ; \mathrm{CDCl} 3) \delta$ $178.5,173.2,136.2,130.0,79.0,52.7,51.8,38.0,37.1,32.8,27.4,26.7,24.0,15.7$.

Acknowledgments: We gratefully acknowledge financial support from the Department of Chemistry at Loughborough University.

Supporting Information: General experimental conditions; optimisation table for the formation of (-)-9a; spectral data for all new compounds and chiral HPLC traces for (-)-9a, (+)-9b, (-)-9b, (-)-9d, (-)-13, (+)-13, (-)-14, (+)-14, (+)-2; crystallographic data or ( \pm )-14; and is available free of charge via Internet at http://pubs.acs.org.

\section{References}

(1) For reviews on the synthetic utility of endoperoxides see, (a) Balci., M. Chem, Rev. 1981, 81, 91; (b) Clennan, E. L. Tetrahedron 1991, 47, 1343. For a review of hydroxy-enones see, (c) Kimber, M. C.; Taylor, D. K. In Trends in Organic Chemistry $2001,9,53$. 

M. C.; Taylor, D. K. J. Org. Chem. 2002, 67, 3142; (c) Avery, T. D.; Fallon, G.; Greatrex, B. W.; Pyke, S. M.; Taylor, D. K.; Tiekink, E. R. T.. J. Org. Chem., 2001, 66, 7955; (d) Avery, T. D.; Taylor, D. K. J. Org. Chem., 2000, 65, 5531. Lactones (e) Brown, R. C.; Taylor, D. K.; Elsey, G. M. Org. Lett., 2006, 8, 463; (f) Zvarec, O.; Avery, T. D.; Taylor, D. K.; Tiekink, E. R. T. Tetrahedron, 2010, 66, 1007; (g) Greatrex, B. W.; Kimber, M. C.; Taylor, D. K.; Fallon, G.; Tiekink, E. R. T. J. Org. Chem., 2002, 67, 5307. Pyrans (h) T. D. Avery; D. Caiazza; J. A. Culbert; D. K. Taylor and E. R. T. Tiekink. J. Org. Chem., 2005, 70, 8344. Tetrahydrofurans (i) Greatrex, B. W.; Kimber, M. C.; Taylor, D. K.; Tiekink, E. R. T. J. Org. Chem., 2003, 68, 4239. Oxidation products (j) Cain, N. M.; Tiekink, E. R. T.; Taylor, D. K. J. Org. Chem., 2012, 77, 3808; (k) Robinson, T. V.; Pederson, D. S.; Taylor, D. K.; Tiekink, E. R. T. J. Org. Chem., 2009, 74, 5093; (I) Greatrex, B. W.; Taylor, D. K.; Tiekink, E. R. T. J. Org. Chem., 2004, 69, 2580; Pyrroles, thiophenes and furans (m) Yang, Y. -K.; Choi, J. -H.; Tae, J. J. Org. Chem., 2005, 70, 6995; (n) Hewton, C. E.; Kimber, M. C.; Taylor, D. K. Tetrahedron Lett., 2002, 43, 3199. Carbenoid insertion (o) Zvarec, O; Avery, T. D.; Taylor, D. K.. J. Org. Chem., 2010, 75, 450; 1,4Diols (p) Spivey, A. C.; Manas, C. G.; Mann, I.. Chem. Commum., 2005, 4426. Bis-epoxides (r) Sing, T. K. M.; Tam, E. K. W. J. Org. Chem., 1998, 63, 1547.

Boyd, J. D.; Foote, C. S.; Imagawa, D. K. J. Am. Chem. Soc., 1980, 102, 3641. anti-inflammatory activity see, (b) Yoon, J. H.; Lim, H. J.; Lee, H. J; Kim, H. -D.; Jeon, R.; Ryu, J. -H. Bioorg. Med. Chem. Lett., 2009, 18, 2179. For reviews on the synthetic approaches to butyrolactones see, (c) Kitson, R. R. A.; Millemaggi, A.; Taylor, R. J. K. Angew. Chem. Int. Ed. 2009, 48, 9426; (d) Seitz, M.; Reiser, O. Curr. Opin. Chem. Biol. 2005, 9, 285; (e) Albrecht, A.; Albrecht, L.; Janecki, T. Eur. J. Org. Chem. 2011, 2747. For recent approaches to the xanthanolides see, (f) Rem, W.; Bian, Y.; Zhang, Z.; Shang, H.; Zhang, P.; Chen, Y.; Yang, Z.; Luo, T.; Tang, Y. Angew. Chem. Int. Ed. 2012, 51, 6984; (g) Matsuo, K.; Ohtsuki, K.; Yoshikawa, K.; Shishido, K.; Yokotani-Tomita, K.; Shindo, M. Tetrahedron 2010, 66, 8407; (h) Kalidindi, S.; Jeong, W. B.; Schall, A.; Bandichhor, R.; Nosse, B.; Reiser, O. Angew. Chem. Int. Ed. 2007, 46, 6361; (i) Kummer, D. A.; Brenneman, J. B.; Martin, S. F. Org. Lett. 2005, 7, 4621. (j) Yokoe, H.; Yoshido, M.; Shishido, K. Tetrahedron Lett. 2008, 49, 3504, (k) Yokoe, H.; Sasaki, H.; Yoshimura, T.; Shindo, M.; Yoshida, M.; Shishido, K.. Org. Lett. 2007, 9, 969

(7) Kornblum, N.; De La Mare, H. J. Am. Chem. Soc. 1951, 73, 881.

(8) For the preparation of 4 see, Smith, C. R; Justice, D. E.; Malpass, J. R. Tetrahedron 1994, 50, 11039.

(9) Toste reported that $\mathrm{CH}_{2} \mathrm{Cl}_{2}$ was the optimum solvent as it exhibited an enhanced rate for the rearrangement (6h) as compared to other solvents. diastereoisomers readily interconvert due to this acidity and that the observed $d r$ is a reflection of the thermodynamic product. 

10.648(3) $\AA, \beta=95.468(4)^{\circ}, V=4026(2) \AA^{3}, Z=8, \mu(\mathrm{Mo}-\mathrm{K \alpha})=0.145 \mathrm{~mm}^{-1}, 20441$ reflections measured, 9762 unique, $R_{\mathrm{int}}=$ $0.0520, R_{1}\left[F^{2}>2 \sigma\left(F^{2}\right)\right]=0.0667, w R_{2}$ (all data) $=0.1841$. Flack parameter $\mathrm{x}=0.41(13)$; both enantiomers present in the asymmetric unit. Space group C2/C was tried but gave a disordered structure. CCDC 907291 contains the supplementary crystallographic data for this paper. These data can be obtained free of charge from The Cambridge Crystallographic Data Centre via www.ccdc.cam.ac.uk/data_request/cif. The crude ${ }^{1} \mathrm{H}$ NMR of the alkylated salt indicated an approximate 4:1 diastereomeric mixture.

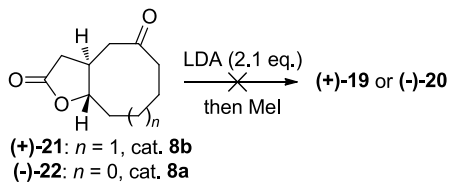

In context, this sequence should be seen as an intermolecular conjugate addition route to the trans-fused lactones, complementary to the intramolecular conjugate addition approach which exclusively give cis-fused lactones; see (a) Burns, A. R.; McAllister, G. D.; Shanahan, S. E.; Taylor, R. J. K. Angew. Chem. Int. Ed. 2010, 49, 5574; (b) Kitson, R. R. A; Taylor, R. J. K.; Wood, J. L. Org. Lett. 2009, 11, 5338; (c) Edwards, M. G.; Kenworthy, M. N.; Kitson, R. R. A.; Perry, A.; Scott, M. S.; Whitwood, A. C.; Taylor, R. J. K. Eur. J. Org. Chem. 2008, 4769; (d) Edwards, M. G.; Kenworthy, M. N.; Kitson, R. R. A.; Scott, M. S.; Taylor, R. J. K. Angew. Chem. Int. Ed. 2008, 47, 1935 Bugarin, A.; Jones, K. D.; Connell, B. T. Chem. Commum. 2010, 46, 1715. 